\title{
Route Recommendation System based on Safety Metrics and Route Profiling
}

\author{
Kripa Sekaran, Priyanka K, Pooja R
}

\begin{abstract}
This project is based on the crime rates happening in our city and the measures taken to curb them to help in strengthening the perception of security in the minds of women and also people who are travelling alone at night. Safe route recommendation is an important part of the field of intelligent transportation, which can provide the guidance of travel mode and travel route for women as well as to travellers. The current route recommendation method has the complexity of urban transports, such as single traffic plan recommendation that often fails to meet the expected requirements. In order to solve the limitation of the one-way vehicle travel method, we propose a safe route recommendation method which includes three modes of transportation, including cars/cabs/auto rickshaws, public transport vehicles, and walking. The routes are represented in different color each denoting a different degree of safety gives user a choice to choose from. The routes/paths are categorized into high, medium and low risk areas. In GI Science, problems related to routing systems have been deeply explored an approach to provide risk score defined by crime rates for generating safe routes This obtained data is then displayed in a map with red, yellow and green patches denoting high, medium and low risk areas respectively. Thus, data are classified by the decision tree (ID3) algorithm. A geospatial repository is used to store tweets related to crime events of the city and the city's street network is converted into graph format which will make the routing and classification mechanism easier. A forecast related to crime events that can occur in a certain place with the collected data was performed. The ID3 classifier classifies each routes into the following labels High, Medium, Low which describes the extent to which the specific route is risky. Our application presents all possible shortest and safe paths between the starting and destination point to the user.
\end{abstract}

Keywords: Classifier, Data Mining, Decision tree algorithm,

\section{INTRODUCTION}

Crime accidents are on a rise all over the world. It is possible to analyze this data to increase the awareness and safety of citizens with the advanced technology and the crime related data are easily available now-a-days. We use the dataset of Tamil Nadu to propose a model for determining the safest path from one location to other.

Revised Manuscript Received on December 12, 2019.

* Correspondence Author

Kripa Sekaran, Assistant Professor, Department of IT, St.Joseph's College of Engineering, Chennai, India. Email: kripasekaran23@gmail.com Priyanka. K, Information Technology, St. Joseph's College of Engineering, Chennai, India.

Pooja. R, Information Technology, St. Joseph's College of Engineering, Chennai, India. Safe route recommendation.

All the crime accidents data sets over the past 15 years that had occurred in Tamil Nadu are collected. The parameters are path ID, path length in $(\mathrm{km})$, risk score along with address and risk extent (high/medium/low). These data sets consist of all the Crimes happened within 15 years. These data sets are taken based on traffic violation to assault, more theft and attempt to murder. Each incident occurred within the city within 15 years is considered in the datasets. These datasets is suited for our daily needs. For this the data is extracted and mined in such a way to suit the need of this application. The path ID used is unique. The path length is calculated from the street profile and also be extracted from address. The risk score is extracted from the number of crime incidents happening in the particular area. For each path the crime rate will be set- if it is below 5 it is considered as low, above 5 is high and around 5 is medium level of risk.

\section{METHODOLOGY}

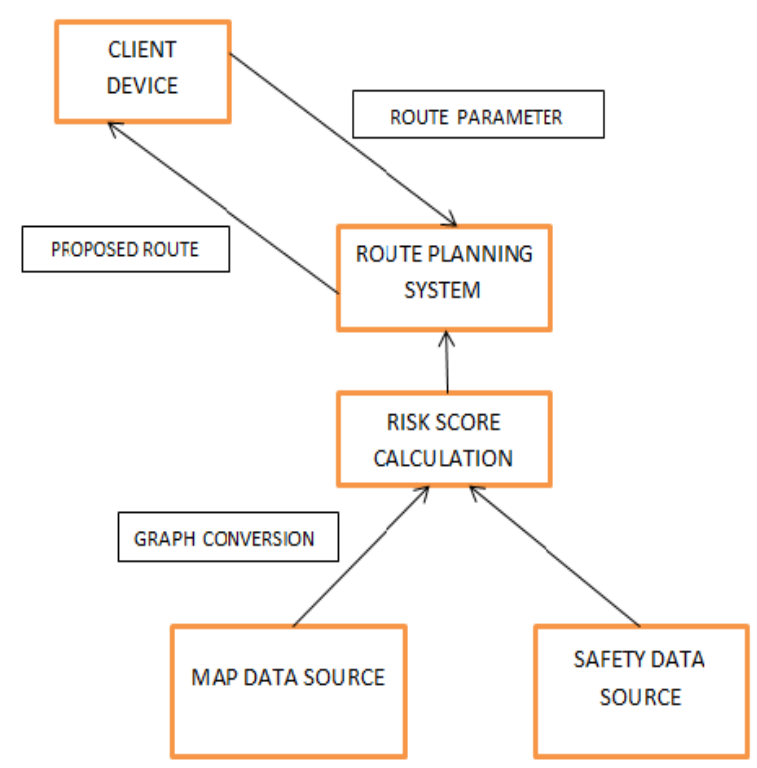

Figure 1: System Overview

Client enters the location (the source and destination) into the application which is an important route parameter. This information is sent to the route planning system, where the model generates all possible routes between the source and destination and sends the location coordinates of the routes to the filter which calculates a weight factor based on the past crime data, distance and number of public transports available on each route. 
Based on the score calculated by the model the routes are classified and represented in different color each denoting a different degree of safety (extent to which that specific route is safe) gives user a choice to choose from.

\section{A.Client Device}

The client device can be any device that is handy and it portable so that it is easy for the users to carry it anywhere they want. Few examples of such devices are, mobile phones, Personal Digital Assistant (PDA), etc.

\section{B.Route Planning System}

Route Planning System is a model in which a risk score is assigned to each edge(path) based on several parameters such as crime data, the total probability of a crime happening at that specific path, traffic intensity, number of surveillance cameras and the spatial constrains imposed by the structure of the city's road network that are collected in real time. Based on the risk score the routes/paths are categorized into high, medium and low risk areas. In our model we use Iterative Dichotomiser 3 or ID3 is an algorithm.

\section{Iterative Dichotomiser 3 or ID3 Algorithm}

Iterative Dichotomiser 3 or ID3 is an algorithm which is used to generate decision tree. First a table is created which contains street number versus all the categories of crimes for a training data set. The basic step in constructing ID3 decision tree is attribute selection. The two mathematical terms used to process attribute selection are Entropy and Information Gain. These 2 terms will be used by the ID3 algorithm to determine which attribute will be selected to become a node of decision tree. All the records in the table are referred as the Collection(c).

TABLE- I: SAMPLE OF TRAINING DATASET

\begin{tabular}{|l|l|l|l|}
\hline Path_ID & $\begin{array}{l}\text { Path_Length } \\
\text { (kilo meters) }\end{array}$ & Risk_Score & $\begin{array}{l}\text { Label } \\
\text { (extent of } \\
\text { risk) }\end{array}$ \\
\hline 1000 & 1.25 & 8.23 & High \\
\hline 1001 & 2.34 & 5.12 & Medium \\
\hline 1002 & 3.12 & 2.23 & Low \\
\hline 1003 & 2.11 & 7.45 & High \\
\hline 1004 & 2.05 & 0.46 & Low \\
\hline 1005 & 3.16 & 2.34 & Low \\
\hline
\end{tabular}

\section{D.Map Data Source}

The city's street network is converted into graph format which consist of nodes $(\mathrm{N})$ and edges $(\mathrm{E})$ which will make the routing and classification mechanism easier and simpler.

\section{E.Safety Data Source}

Dataset contains criminal incidents over few years that have occurred in the city. The various parameters considered are date of the incidence, the time and address of occurrence of the crime, the description of the crime, the category of the crime, action taken against the crime and geographical location of the occurrence of the incidence. The dataset consists of all the crimes that have occurred within few years. These crimes range from petty traffic violations to assault and attempt to murder. Each incident within the city that occurred within the period of time is considered in the dataset.

\section{PROPOSED APPROACH TO FIND SAFE ROUTE}

\section{A.Input from the User}

The user must first log into his/her own profile/account which contains all the user's details such as phone number, permanent address, gender, etc. Then the user will provide input in the form of two locations -source and destination respectively. From source to destination there maybe multiple possible routes. For each route the risk factors are analyzed and a risk score is calculated, based on which the safe routes are profiled and recommended to the user. Multiple possible paths will be analyzed for safety.

\section{B.Constructing the Classifier}

Based on the source and destination location entered by the user, all the possible routes and each route constituting a set of paths are analyzed and the risk total risk score for each route is calculated and the ID3 classifier classifies each routes into the following labels High, Medium, Low which describes the extent to which the specific route is risky.

\section{C.Risk Score calculation}

The safest route can be determined from the safety of all of its paths. The unsafe streets in that route will be analyzed further for risk score. Risk score is the risk involved in a particular street. [12] Risk weight for a street is calculated by summing up the multiplication of the count of occurrences of nth crime with the crime factor for that crime. The value of the crime factor is proportional to the punishment prescribed for the crime. More heinous the crime higher is the crime factor. Calculation of risk score of path-i:

Risk score of path- $i=\Sigma$ count $*$ Crime factor Where, Crime factor $=$ the intensity of the crime. The route with the minimum amount of risk will be the safest route. The user will have the option to share the safest route on social media to let well-wishers know the route he will be taking.

\section{DRAWBACKS OF EXISTING SYSTEM}

1. The existing idea suggests only one safe route without giving any other choice to the user.

2. The system recommends route based on past crime report including the frequency in which the crime occur, but it not based on any real time data.

3. The recommendation system takes only crime occurrence as the major factor determine safe route, where as it does not take any other factors (that are crucial to determine road safety) into consideration. 


\section{RESULTS AND DISCUSSION}

According our proposed system, based on the calculated risk score the destination paths are classified and the best routes are presented to the user, the various features of our system are as follows:

1) Our proposed recommendation system takes not only crime occurrence as the major factor determine safe route, but also take other factors (that are crucial to determine road safety) such as availability of public transport, demographic data etc., into consideration.

2) The proposed system recommends route not only based on past crime report including the frequency in which the crime occur, but also based on the real time data.

3) Our proposed route recommendation system, recommends a number of routes represented in different color each denoting a different degree of safety (extent to which that specific route is safe) gives user a choice to choose from.

4) The system gives perfect visual representation of the extent to which the recommended route is safe to the user.

\section{CONCLUSION}

An application of data mining, which will determine the safest route from source to destination based on ID3 decision tree algorithm, has been presented. The safety of the user can be assured if this application is used. The Application minimizes the risk involved while travelling through a particular area based on risk score. Social support through social media or messaging and additional facilities will also be provided to ensure that close ones know where the particular user will travel from. In future scope, the data can be analyzed to reinforce security in the areas within the city which are less secure or risk prone. In another perspective, profiling of criminals can be done based on past crime reports. For this, repetitive offenders can be mapped to their corresponding categories of crimes or their areas of activity to help solve future cases. In addition, safer areas from real estate perspective can be determined. Also verified users can travel together for common destinations.

\section{REFERENCES}

1. Mata, F., Torres-Ruiz, M., Guzmán, G., Quintero, R., Zagal-Flores, R., Moreno-Ibarra, M., \& Loza, E. (2016). A mobile information system based on crowd-sensed and official crime data for finding safe routes: A case study of mexico city. Mobile Information Systems, 2016.

2. EHSANI, Pouya Johnathon; BRUSS, Christopher Bayan; KHODADAD, Jian Khadem. Route planning system and methodology which account for safety factors. U.S. Patent Application No 14/205,495, 2015.

3. TORNBERG, Goteborg SE. Identifications Optimization Generation. 2012.

4. SPEIER, Gary J.; LUNDBERG, Steven W.; ARMITAGE, Benjamin C. System and method for travel route planning using safety metrics. U.S. Patent No 8,315,792, 2012.

5. D Delling, A V Goldberg, T Pajor et al., "Customizable route planning[C]", International Conference on Experimental Algorithms, pp. 376-387, 2011

6. Tate, Edward D., and Charles A. Massoll. "Route planning system for vehicles." U.S. Patent No. 9,008,955. 14 Apr. 2015.

7. Makarova, Irina, Anton Pashkevich, and Ksenia Shubenkova. "Safe Routes as One of the Ways to Reduce the Number of Road Accidents Victims." Scientific And Technical Conference Transport Systems Theory And Practice. Springer, Cham, 2017.
8. CUI, Ge; LUO, Jun; WANG, Xin. Personalized travel route recommendation using collaborative filtering based on GPS trajectories. International journal of digital earth, 2018, 11.3: 284-307.

9. Utamima, Amalia, and Arif Djunaidy. "Be-safe travel, a web-based geographic application to explore safe-route in an area." AIP Conference Proceedings. Vol. 1867. No. 1. AIP Publishing, 2017

10. J H Hu, Z Huang, J. Deng, "A Hierarchical Path Planning Method Using the Experience of Taxi Drivers [J]", Procedia - Social and Behavioral Sciences, vol. 96, pp. 1898-1909, 2013.

11. S. Deerwester, S. Dumais, T. Landauer, G. Furnas, and R. Harshman, "Indexing by Latent Semantic Analysis," J. Am. Soc. Inf. Sci., vol. 41, no. 6, pp. 391-407, 199

12. Sarang Tarlekar et al, / (IJCSIT) International Journal of Computer Science and Information Technologies, Vol. 7 (3) , 2016, 1536-1540

\section{AUTHORS PROFILE}

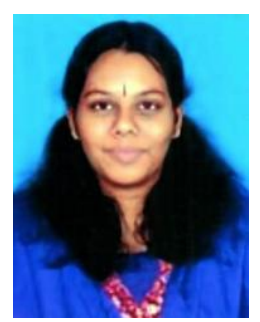

Kripa Sekaran, Assistant Professor, Department of IT had completed her B.Tech in Information Technology from Jeppiaar Engineering college, Chennai and M.E in Computer science engineering from SSN College of Engineering Chennai. The author has published papers in renowned journals like International Journal of Applied Engineering and Research and IEEE digital library. The author's research interest includes machine learning, software engineering and big data analytics.

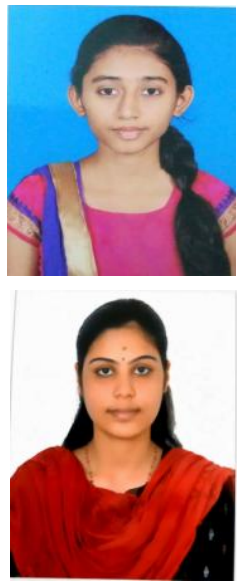

Priyanka. K, holds B.Tech. in Information Technology, St.Joseph's college of Engineering, Chennai. Represented Tamil Nadu at $20^{\text {th }}$ National Children's science congress held at Banaras Hindu University (Varanasi). The author's research interest includes machine learning and data analytics.

Pooja. R, holds B.Tech. in Information Technology, St.Joseph's college of Engineering, Chennai. The author's research interest includes machine learning, data mining and data analytics. 